\title{
LDHA promotes tumor metastasis by facilitating epithelial-mesenchymal transition in renal cell carcinoma
}

\author{
JUPING ZHAO* ${ }^{*}$ XIN HUANG ${ }^{*}$, ZHAOPING XU, JUN DAI, HONGCHAO HE, YU ZHU and HAOFEI WANG \\ Department of Urology, Ruijin Hospital, Shanghai Jiao Tong University School of Medicine, Shanghai 200025, P.R. China
}

Received February 10, 2017; Accepted August 11, 2017

DOI: $10.3892 / \mathrm{mmr} .2017 .7637$

\begin{abstract}
Previous studies have indicated that high expression of lactate dehydrogenase A (LDHA) exists in many human cancers. Recently, several reports showed that silencing or inhibition of LDHA could suppress metastasis of human cancer including renal cell carcinoma (RCC). However, the mechanism remains unknown. The role of LDHA in RCC migration and invasion was investigated using immunohistochemistry, western blotting, Transwell and scratch assays, and in vivo experiment. The influence of LDHA on the Warburg effect was also investigated by LDHA activity and lactate production assay. LDHA was overexpressed in RCC tissues and predicted a worse survival following renal resection. Correlation analysis demonstrated that LDHA was negatively correlated with E-cadherin and positively with $\mathrm{N}$-cadherin. Experimentally, both in vivo and in vitro experiments found downregulation of LDHA suppressed RCC cells migration and invasion by inhibiting EMT. In addition, results indicated LDHA could promote the Warburg effect. Further research presented that the LDHA inhibitor, oxamate, suppressed tumor metastasis by inhibiting LDHA activity and EMT. These results demonstrated that LDHA mediates tumor metastasis by promoting EMT in RCC, suggesting that LDHA could be a promising therapeutic target for RCC therapy.
\end{abstract}

Correspondence to: Dr Haofei Wang, Department of Urology, Ruijin Hospital, Shanghai Jiao Tong University School of Medicine, 197 Ruijin 2nd Road, Shanghai 200025, P.R. China

E-mail:wanglog@sina.com

*Contributed equally

Abbreviations: RCC, renal cell carcinoma; siRNA, small interfering RNA; EMT, epithelial-mesenchymal transition; FFPE, formalin-fixed paraffin-embedded; US, ultrasound; CT, computed tomography; MRI, magnetic resonance imaging; SD, standard deviation; OS, overall survival; DFS, disease-free survival; T, tumor tissue; NT, corresponding non-tumor tissue

Key words: epithelial-mesenchymal transition, metastasis, LDHA, renal cell carcinoma

\section{Introduction}

Renal cell carcinoma (RCC) is a common cancer in the world, which accounts to $\sim 85 \%$ of renal malignancy (1). The incidence of RCC varies in different region (2). Most of new cases occur in developed countries, such as Europe and North America (3). 20-30\% of patients with RCC are diagnosed at advanced stage with metastasis, and $30 \%$ of those diagnosed at early stage will eventually developed metastasis after resection (4). Due to metastasis and resistance to chemotherapy and radiotherapy, RCC has been reported to carry a poor prognosis (5). This emphasizes the importance to develop new therapeutic approaches to improve prognosis and quality of life of patients.

Aerobic glycolysis, also known as the Warburg effect, is featured by the increased glucose utilization and lactate production in tumor cells even at normal oxygen concentrations (6). Recent studies demonstrated that the Warburg effect serve an important role in tumor development and progression $(7,8)$. Numerous studies reported that the expression of genes involved in energetic metabolism displayed heterogeneity in RCC, suggesting that the Warburg effect may participate in the development of RCC $(9,10)$. However, the function and mechanism of the Warburg effect in RCC remains unknown. Lactate dehydrogenase A (LDHA), a key enzyme in glycolysis, promotes the shift from pyruvate to lactate. Previous studies showed that the level of LDHA is upregulated in many human cancers $(11,12)$. Another group of investigators had observed that silencing or inhibition of LDHA suppresses tumor growth and metastasis of human cancer (13-15). All of this evidence implies the underlying role of LDHA in cancer development and progression (16). Recent studies manifested that the expression of LDHA was up-regulated in RCC, and the high level of LDHA was correlated with poor differentiation of tumor cells and poor prognosis of RCC patients $(17,18)$. However, the mechanism remains unknown.

Epithelial-mesenchymal transition (EMT), a vital event in invasion and metastasis of human cancer, allows tumor cells to lose the epithelial phenotype and obtain mesenchymal phenotype, which makes tumor cells acquire the ability of motility $(19,20)$. The role of EMT in tumor metastasis has been widely accepted (21-23). Downregulation of E-cadherin, which is the primary marker of EMT process, is regarded as signaling an extremely poor prognosis. Various 
research has demonstrated that dysregulated elements leading to EMT can be potential targets in malignant tumor. Piva et al (24) reported that EMT contributed to RCC development and progression. Jiang et al (25) reported that LDHA was elevated in muscle-invasive bladder cancer, promoting malignant progression via activation of EMT. The above findings prompted us to investigate the effects of LDHA in EMT process in RCC.

In order to decipher the molecular mechanisms in the present study, the authors examined the expression of LDHA in RCC patients, and then analyzed the correlation between the LDHA and the clinicopathological parameters of patients with RCC. Furthermore, the relationship between LDHA expression and EMT markers was evaluated. Different approaches were employed to explore the role of LDHA expression in EMT process and metastasis of tumor cells. Besides, LDHA specific inhibitor oxamate was used to verify the function of LDHA in RCC. Finally, an orthotopic renal xenograft model was established to determine the function of LDHA on tumor metastasis in vivo. The present study elaborated the molecular mechanisms of LDHA in EMT and progression of RCC, and could represent a more promising strategy against RCC.

\section{Materials and methods}

Ethical review. The protocol of the research project had been approved by the Ethics Committee of the Ruijin Hospital, Shanghai Jiaotong University (Shanghai, China) and it conformed to the provisions of the Declaration of Helsinki. The patients' informed consents were all obtained.

Tissue specimens and immunohistochemical staining analysis (IHC). All formalin-fixed paraffin-embedded RCC tissue specimens and normal renal tissue of RCC patients who underwent radical nephrectomy or nephron-sparing surgery were obtained from the Department of Urology, Ruijin Hospital, Shanghai Jiaotong University School of Medicine (Shanghai, China) between 2009 and 2015. The diagnosis of RCC was made by at least two contrast-enhanced imaging studies [ultrasound (US), computed tomography (CT) and magnetic resonance imaging (MRI)], and confirmed by US-assisted fine-needle biopsy. None of patients had received chemotherapy or radiotherapy before the operation. Follow up was performed from the date of tumor resection until May 2015 or until patient mortality. All clinicopathological parameters were collected from the medical records of the hospital.

Specimens were stained at $4^{\circ} \mathrm{C}$ overnight with LDHA polyclonal antibody (cat. no. SC-27231; dilution, 1:200; Santa Cruz Biotechnology, Inc., Dallas, TX, USA), E-cadherin polyclonal antibody (cat. no. 3195; dilution, 1:200), N-cadherin polyclonal antibody (cat. no. 13116; dilution, 1:200), vimentin polyclonal antibody (cat. no. 5741; dilution, 1:200) and Snail polyclonal antibody (cat. no. 3879; dilution, 1:200) all obtained from Cell Signaling Technology, Inc. (Danvers, MA, USA). A biotinylated secondary antibody (cat. no. 10K06A; dilution, 1:100; ZSGB-BIO; OriGene Technologies, Inc.) was used to detect the primary antibody at $37^{\circ} \mathrm{C}$ for $40 \mathrm{~min}$. A score was calculated via multiplying the extent of staining $(1,0-5 \% ; 2,5-25 \%$; $3,26-50 \% ; 4,50-75 \% ; 5,75-100 \%)$ by the staining intensity ( 0 , negative; 1 , weak; 2 , medium; 3 , strong). For the purpose of statistical analyses, tumors with a final staining score of $>3$ were considered as high expression.

Western blot assay. Cells and tissues were lysed in lysis buffer containing protease inhibitor cocktail (50 mM Tris $\mathrm{pH} 7.5$, $150 \mathrm{mM} \mathrm{NaCl}, 1 \%$ TritonX-100 and $5 \mathrm{mM}$ ethylenediaminetetraacetic acid). Protein concentration was determined using a Bio-Rad protein assay system (Bio-Rad Laboratories, Inc., Hercules, CA, USA). Western blotting was performed following the routine protocol. Signals were detected on X-ray film using the ECL detection system (Pierce; Thermo Fisher Scientific, Inc., Waltham, MA, USA). The relative protein levels were calculated based on $\beta$-actin as the loading control.

Cell culture and small interfering (si)RNA transfection. Human RCC cell lines 786-O, OS-RC-2, Caki-1, A498, HK-2 and $\mathrm{ACHN}$ were obtained from the Institute of Biochemistry and Cell Biology, Chinese Academy of Sciences (Shanghai, China) and were preserved in our institute. All the cells were cultured in RPMI-1640 medium (HyClone Laboratories; GE Healthcare Life Sciences, Chalfont, UK) with $10 \%$ fetal bovine serum (Gibco; Thermo Fisher Scientific, Inc.), $100 \mathrm{U} / \mathrm{ml}$ penicillin and $100 \mu \mathrm{g} / \mathrm{ml}$ streptomycin and maintained at $37^{\circ} \mathrm{C}$ with $5 \% \mathrm{CO}_{2}$. Transfection of specific siRNAs targeting LDHA (Shanghai GenePharma Co., Ltd., Shanghai, China) and negative control (NC)-siRNA into RCC cells was performed using Lipofectamine ${ }^{\circledR} 2000$ reagent (Invitrogen; Thermo Fisher Scientific, Inc.) following the manufacturer's protocol. Following this, the interference efficiency was determined by western blotting. ACHN cells with stable knockdown of LDHA and their control line were established using the lentiviral transduction system (Santa Cruz Biotechnology, Inc.) (26).

LDHA activity, glucose and lactate measurement. RCC cells $\left(1 \times 10^{6}\right)$ were transfected with siRNAs or cultured with $30 \mathrm{mM}$ oxamate (Sigma-Aldrich; Merck KGaA, Darmstadt, Germany) and prepared for LDHA activity, lactate production and glucose utilization assay. LDHA activity and lactate production assay were performed using the Lactate Dehydrogenase Activity Assay kit (cat. no. MAK066) and Lactate Assay kit (cat. no. MAK064; Sigma-Aldrich; Merck KGaA) according to the manufacturer's protocol. Glucose concentration in the media was measured using colorimetric glucose assay kit (BioVision, Milpitas, CA, USA) and normalized according to cell number (27).

Invasion and migration assay. The scratch migration assay was performed in 24-well plate, and cells were transfected with control or LDHA siRNA, or cultured with $30 \mathrm{mM}$ oxamate. Cells were scratched using a tip of sterile $200 \mu$ l pipette in each well following $24 \mathrm{~h}$. The plates were washed twice with $\mathrm{PBS}$, and then incubated at $37^{\circ} \mathrm{C}$ in $5 \% \mathrm{CO}_{2}$. Wound width was monitored at various time points and quantified by the ratio of gap distance at 24 or $48 \mathrm{~h}$ to that at $0 \mathrm{~h}$. The experiment was conducted in triplicate. The migration and invasion assay were both performed using Transwell (Corning Incorporated, Corning, NY, USA) in 24-well plate. Filters coated with Matrigel (BD Biosciences, Franklin Lakes, NJ, USA) were used for invasion assay. At $48 \mathrm{~h}$ following RNA interference, 
cell suspension ( $1 \times 10^{5}$ cells in $200 \mu 1$ serum-free medium) was plated into the upper chamber, while the lower chamber was filled with $500 \mu 1$ R PMI (HyClone Laboratories; GE Healthcare Life Sciences) containing 10\% FBS (Gibco; Thermo Fisher Scientific, Inc.). Following incubation at $37^{\circ} \mathrm{C}$ for $24 \mathrm{~h}$, tumor cells adhere to the lower surface of the membrane were fixed with methanol and stained with crystal violet. The staining cells were photographed and counted using Image $\mathbf{J}$ software.

In vivo metastasis analysis. All procedures for animal experiments were performed in accordance with the Guide for the Care and Use of Laboratory Animals (NIH publications Nos. 80-23, reversed 1996) and according to the Ethical Guidelines for animal experiments of the Ruijin Hospital, Shanghai Jiaotong University (Shanghai, China). The orthotopic renal xenograft model was established in previous research (26). RCC cells were suspended in $20 \mu \mathrm{l}$ RPMI-1640/Matrigel (1:1; R\&D Systems, Inc., Minneapolis, MN, USA) and inoculated into the right subrenal capsule of kidneys of nude mice. At 7 weeks, the two group of ten mice were sacrificed by cervical dislocation and the lungs were removed and embedded in paraffin for evaluating the frequency of metastasis, serial sections from lung were stained with hematoxylin and eosin $(H \& E)$ and then screened independently by two researchers who were blinded to the treatment.

Statistical analysis. All data from at least three independent experiments was presented as mean \pm standard deviation and statistically analyzed using SPSS software (version 18.0; SPSS, Inc., Chicago, IL, USA). Statistical analysis was performed by Student's t-test. The rate and constituent ratio were compared by the Chi-square test. Kaplan-Meier survival curve was used to analyze patient's overall survival (OS) rates and disease-free survival (DFS) rates, and the differences were compared using the log-rank test. $\mathrm{P}<0.05$ was considered to indicate a statistically significant difference.

\section{Results}

Association of expression of LDHA with poor prognosis in $R C C$. To verify the expression of LDHA in RCC, the authors examined the level of LDHA in 43 RCC and corresponding non-cancerous renal (CNR) tissues using immunohistochemical staining and western blotting. Subsequent analyses revealed that LDHA was upregulated in 55.8\% (24/43) of RCC patients compared with those of CNR tissues $(\mathrm{P}=0.016$; Fig. 1A and $\mathrm{B}$ ). In addition, western blotting implied that LDHA expression level was enhanced in 786-O and ACHN cells compared to OS-RC-2, Caki-1, A498 and HK-2 cells (Fig. 1C). Therefore, 786-O and ACHN cells were employed in the subsequent experiments. The above observations suggested that LDHA was upregulated in RCC.

In order to investigate the relationship between LDHA expression and clinicopathological characteristics of RCC, the clinical data of $43 \mathrm{RCC}$ patients were retrospectively analyzed. Results showed that increased LDHA expression was significantly correlated with lymph node metastasis $\left(\chi^{2}=4.449, \mathrm{P}=0.025\right)$, distant metastasis $\left(\chi^{2}=5.044, \mathrm{P}=0.035\right)$, and tumor size $\left(\chi^{2}=6.894, \mathrm{P}=0.009\right.$; Table I). The OS rate and DFS rate between the two groups had significant
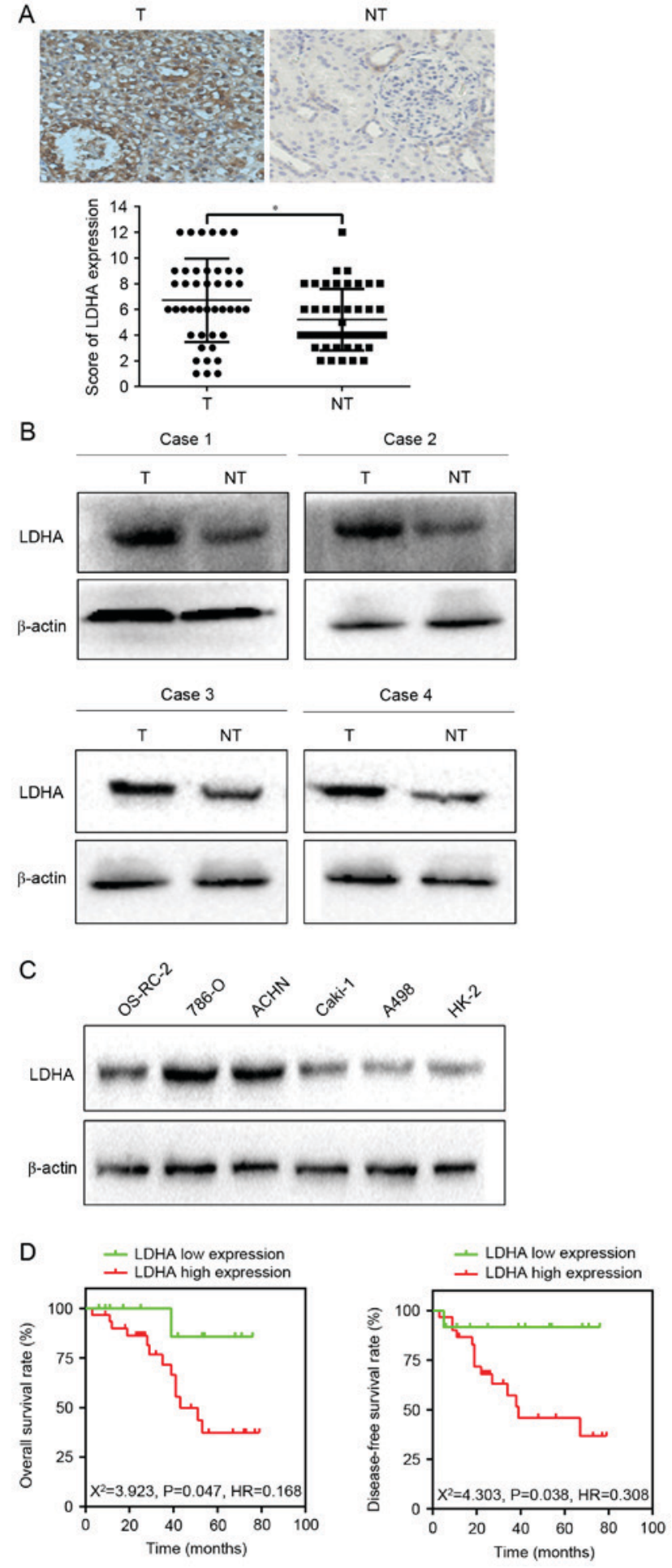

Figure 1. LDHA expression and its prognostic value in RCC patients. (A) Representative images of LDHA immunohistochemical staining in RCC tissues and the corresponding non-tumor tissues (magnification, x200). (B) Western blotting showed that LDHA protein had high expression in RCC tissue. (C) Western blotting showed that LDHA expression levels were higher in 786-O and ACHN cells compared to OS-RC-2, Caki-1, A498 and HK-2 cells. (D) Kaplan-Meier curves reported that the prognosis of RCC patients with higher LDHA expression was poorer than that with lower LDHA expression $(\mathrm{P}<0.05, \log -$ rank test $)$. Data are presented as the mean \pm standard deviation. "P<0.05 vs. control. LDHA, lactate dehydrogenase A; RCC, renal cell carcinoma; T, tumor tissue; NT, non-tumor tissue.

differences, as evidenced by the Kaplan-Meier survival curve and the log-rank test $\left(\mathrm{OS}, \chi^{2}=3.923, \mathrm{P}=0.0476\right.$; DFS, $\chi^{2}=4.245$, 
Table I. Association of LDHA expression with clinicopathological factors of 43 RCC patients.

LDHA expression

\begin{tabular}{|c|c|c|c|c|}
\hline \multirow{2}{*}{ Clinicopathological parameters } & \multirow{2}{*}{ Cases } & & & \multirow{2}{*}{ P-value } \\
\hline & & Low $(n=12)$ & $\operatorname{High}(\mathrm{n}=31)$ & \\
\hline Age (years) & & & & 0.836 \\
\hline$\leq 60$ & 19 & 5 & 14 & \\
\hline$>60$ & 24 & 7 & 17 & \\
\hline Gender & & & & 0.099 \\
\hline Male & 20 & 8 & 12 & \\
\hline Female & 23 & 4 & 19 & \\
\hline Histological grade & & & & 0.497 \\
\hline Well & 27 & 9 & 18 & \\
\hline Moderate and poor & 16 & 3 & 13 & \\
\hline Tumor size & & & & $0.009^{\mathrm{a}}$ \\
\hline$\leq 5 \mathrm{~cm}$ & 22 & 10 & 12 & \\
\hline$>5 \mathrm{~cm}$ & 21 & 2 & 19 & \\
\hline Lymph node metastasis & & & & $0.025^{\mathrm{b}}$ \\
\hline Positive & 10 & 0 & 10 & \\
\hline Negative & 33 & 12 & 21 & \\
\hline Distant metastasis & & & & $0.035^{\mathrm{b}}$ \\
\hline Positive & 14 & 1 & 13 & \\
\hline Negative & 29 & 11 & 18 & \\
\hline
\end{tabular}

It had been analyzed that increased LDHA expression was significantly correlated with tumor size $\left(\chi^{2}=6.894 ; \mathrm{P}=0.009\right)$, lymph node metastasis $\left(\chi^{2}=4.449 ; \mathrm{P}=0.025\right)$ and distant metastasis $\left(\chi^{2}=5.044 ; \mathrm{P}=0.035\right) .{ }^{\mathrm{a}} \mathrm{P}<0.01 ;{ }^{\mathrm{b}} \mathrm{P}<0.05$. LDHA, lactate dehydrogenase $\mathrm{A}$.

$\mathrm{P}=0.0394$; Fig. 1D). All of the data indicated that LDHA might have an important role in the development of RCC.

Downregulation of LDHA inhibited migration and invasion of RCC cells. Following this, the authors hypothesized about the functional role of LDHA in tumor cells. Western blotting revealed that LDHA protein levels were obviously decreased in 786-O and ACHN cells that transfected with LDHA-siRNA, compared with their control groups respectively (Fig. 2A). Then assays were performed to investigate the role of LDHA in migration of invasion of RCC cells. The Transwell assay indicated that downregulation of LDHA significantly decreased the number of 786-O and ACHN cells crossing the membrane compared to the control group, as presented in Fig. $2 \mathrm{~B}(\mathrm{P}<0.01$ for $786-\mathrm{O}$ and $\mathrm{ACHN}$ ). Matrigel invasion assay showed that the number of LDHA-siRNA cells crossing Matrigel-coated membrane significantly decreased compared to the control group ( $\mathrm{P}<0.01$ for $786-\mathrm{O}$ and $\mathrm{ACHN}$, Fig. $2 \mathrm{C})$. The same conclusions were reached following the scratch migration assay $(\mathrm{P}=0.0374$ for $786-\mathrm{O}$ and $\mathrm{P}=0.0246$ for $\mathrm{ACHN}$, Fig. 2D and E). These data illustrated that the low-expression of LDHA significantly suppresses the ability of migration and invasion of RCC cells.

Knockdown of LDHA inhibits tumor metastasis in vivo. To determine the function of LDHA on tumor metastasis in vivo, an orthotopic renal xenograft model was established in the present research. At 7 weeks, the mice were sacrificed and the pulmonary metastasis foci were counted. Of note, average metastatic nodules of lung were dramatically decreased in the LDHA-siRNA group, compared to NC siRNA groups $(\mathrm{P}<0.033$; Table II). Hematoxylin and eosin (H\&E) staining of serial sections of lung were used to identify metastasis nodules (Fig. 2F). These results suggested that LDHA is a critical factor inhibiting RCC metastasis in vivo.

LDHA promoted the Warburg effect in RCC cells. Accumulating evidence has demonstrated that the Warburg effect played an important role in the development of tumor, and may have potential clinical applications (24). The influence of LDHA on the Warburg effect in RCC cells was also investigated. In the present study, the effect of LDHA on glucose utilization and lactate production was assessed. Significant decreasing in glucose utilization $(\mathrm{P}=0.0019$ for $786-\mathrm{O}$ cells and $\mathrm{P}=0.035$ for ACHN cells) and lactate production $(\mathrm{P}<0.01$ for $786-\mathrm{O}$ cells and $\mathrm{P}=0.019$ for ACHN cells) in LDHA-siRNA RCC cells were observed (Fig. $3 \mathrm{~A}$ and $\mathrm{B}$ ). These data indicated that LDHA could affect the Warburg effect, which influences the development of RCC.

LDHA suppressed epithelial phenotype and promoted mesenchymal transition. Limited investigation attempted to elucidate that LDHA could promote EMT in muscle-invasive bladder cancer (25). In the current study, retrospective analyses showed that LDHA expression was related to tumor 
Table II. Incidence of metastatic tumors of the lung.

\begin{tabular}{lccc}
\hline Group & Numbers & Incidence of renal cell carcinoma & Metastatic rates \\
\hline LDHA-siRNA & 10 & $10 / 10(100 \%)$ & $5 / 10(50 \%)$ \\
NC siRNA & 10 & $10 / 10(100 \%)$ & $10 / 10(100 \%)$ \\
P-value & & & 0.033 \\
\hline
\end{tabular}

LDHA, lactate dehydrogenase A; NC, negative control; siRNA, small interfering RNA.

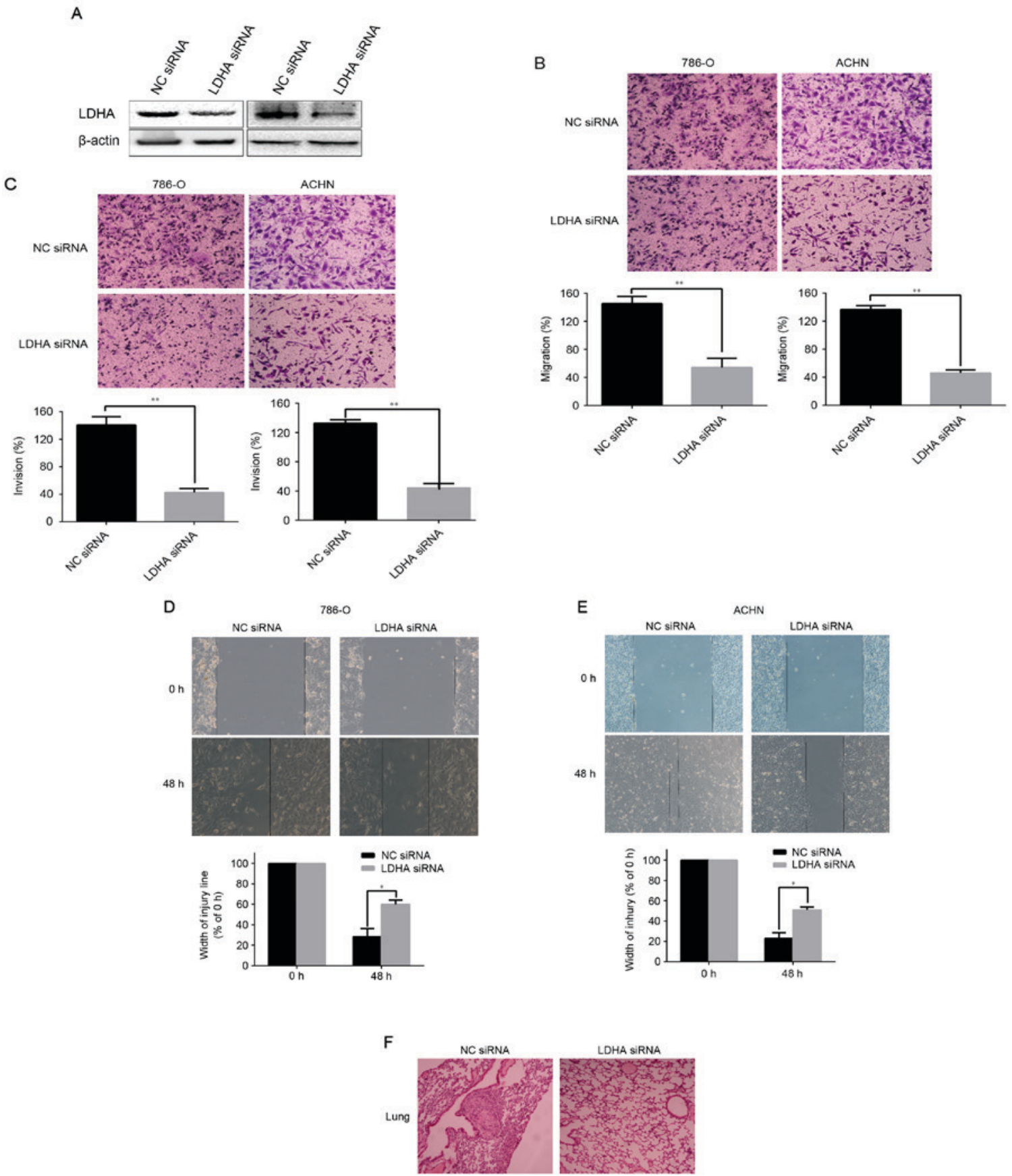

Figure 2. Downregulation of LDHA Inhibited the migration and invasion of RCC cells in vitro. (A) LDHA protein levels were obviously decreased in 786-O and ACHN cells by LDHA-siRNA, compared with their control groups respectively. (B and C) Migration and invasion ability of RCC cells transfected with control siRNAs or LDHA siRNA were determined by uncoated or Matrigel-coated Transwell assay in 786-O and ACHN cells respectively. (D and E) Migration of RCC cells transfected with control siRNAs or LDHA siRNA were measured by scratch assay in 786-O and ACHN cells respectively. (F) Serial sections of lung of RCC mouse models were stained with hematoxylin and eosin stain to identify pulmonary metastatic nodules. Representative images were shown (magnification, $\mathrm{x} 100$ ). Data are presented as mean \pm standard deviation. ${ }^{*} \mathrm{P}<0.05,{ }^{* *} \mathrm{P}<0.01$ vs. control. LDHA, lactate dehydrogenase A; RCC, renal cell carcinoma; siRNA, small interfering RNA; NC, negative control. 
A

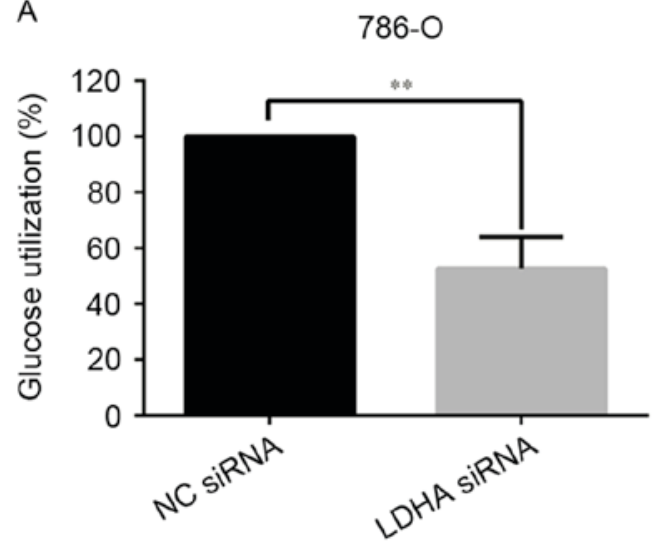

B

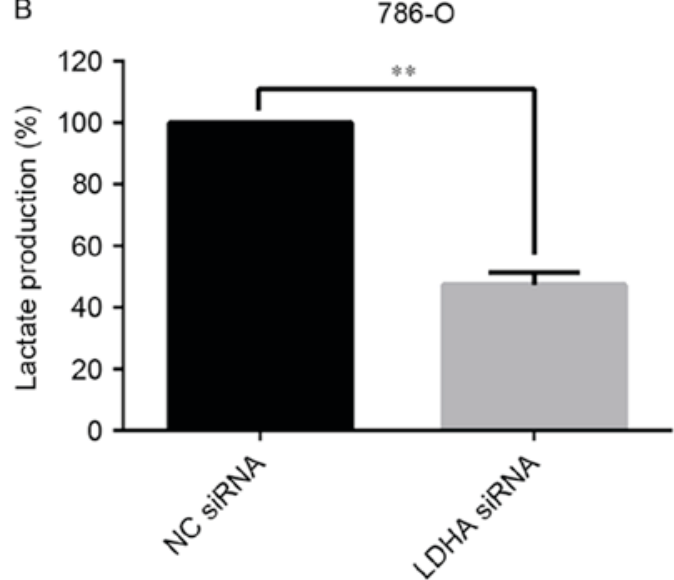

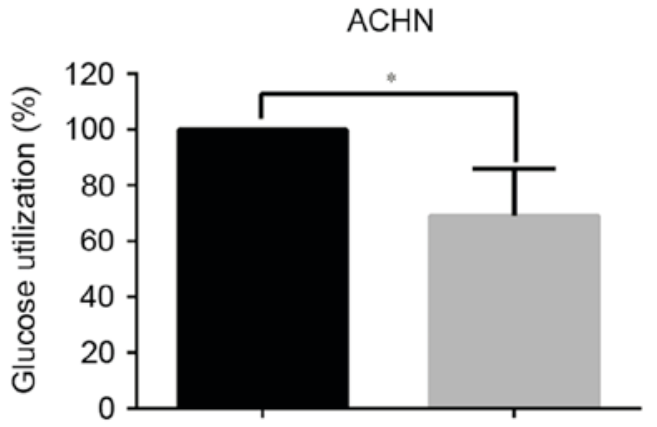
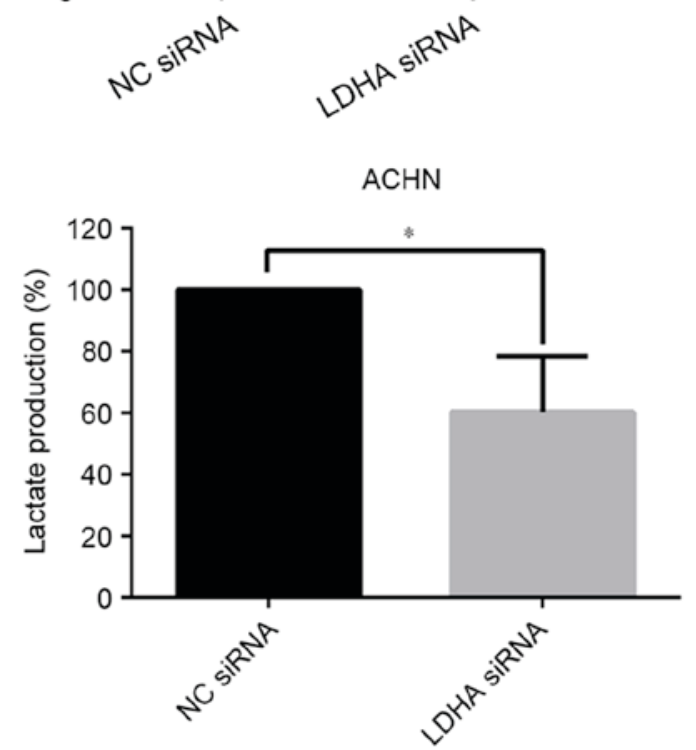

Figure 3. Regulation of glucose utilization and lactate production by downregulating LDHA expression. (A) The glucose utilization was decreased by LDHA siRNA compared with control. (B) The lactate production was reduced by LDHA siRNA compared with control. Data are presented as mean \pm standard deviation. ${ }^{*} \mathrm{P}<0.05,{ }^{* *} \mathrm{P}<0.01$ as indicated. LDHA, lactate dehydrogenase A; siRNA, small interfering RNA; NC, negative control.

metastasis. Therefore, the authors ask whether LDHA expression was related to EMT process. To this end, the correlation between the expression of LDHA and EMT markers was tested. The results of IHC indicated that the level of reduction of E-cadherin $(\mathrm{P}=0.0498)$ and elevation of $\mathrm{N}$-cadherin $(\mathrm{P}=0.0286)$ and vimentin $(\mathrm{P}=0.033)$ were dramatically correlated with the upregulation of LDHA (Fig. 4A). In addition, a negative correlation between LDHA expression and E-cadherin expression and a positive correlation between LDHA expression and N-cadherin were explored using Pearson's correlation coefficient (Fig. 4B). Western blotting of RCC tissues revealed that the downregulation of LDHA was negatively associated with epithelial phenotype and positively associated with mesenchymal phenotype, which was consistent with the IHC observations (Fig. 4C).

In order to substantiate the hypothesis that LDHA may inhibit EMT, we further tested the EMT makers in LDHA-siRNA cells and the control group, which yielded similar results. An increased level of E-cadherin was observed as a response to the treatment of LDHA-siRNA in RCC cells. On the contrary, $\mathrm{N}$-cadherin and vimentin were attenuated in LDHA-siRNA cells compared to the control group (Fig. 4D). Furthermore, downregulation of LDHA suppressed the expression of EMT inducer Snail (Fig. 4C and D). These findings make clear that EMT process plays an important role in LDHA induced metastasis in RCC cells.
Oxamate suppressed tumor metastasis by inhibiting LDHA activity and EMT process. Considering the clinical application of target therapy, the authors treated the RCC cells with oxamate to investigate the contribution of LDHA inhibitor to LDHA activity and LDHA-driven EMT. The concentration of oxamate was $30 \mathrm{mM}$ according to the authors' previous study. The results demonstrated that oxamate significantly decreased LDHA activity, glucose utilization, and lactate production of RCC cells compared with the control group $(\mathrm{P}<0.01$; Fig. 5A). As illustrated in Fig. 5B, oxamate apparently inhibited the ability of migration and invasion of 786-O RCC cells by Transwell assays compared to the control group $(\mathrm{P}<0.01)$. The scratch migration assay, in line with Transwell assays confirmed that oxamate significantly suppressed the migration of 786-O RCC cells ( $\mathrm{P}<0.05$; Fig. 5C). The above observations increasingly support the notion that LDHA promote RCC metastasis by mechanisms orchestrated by EMT.

\section{Discussion}

RCC is the leading cause of cancer-death worldwide. Surgical treatment at early stage is the only curative therapy for RCC patients. Unfortunately, due to high frequency of intrarenal and extrarenal metastasis, most of RCC patients are diagnosed at the advanced stage and no suitable to receive the curative therapy, which leads to poor prognosis of RCCs. Hence, it is 
A

T

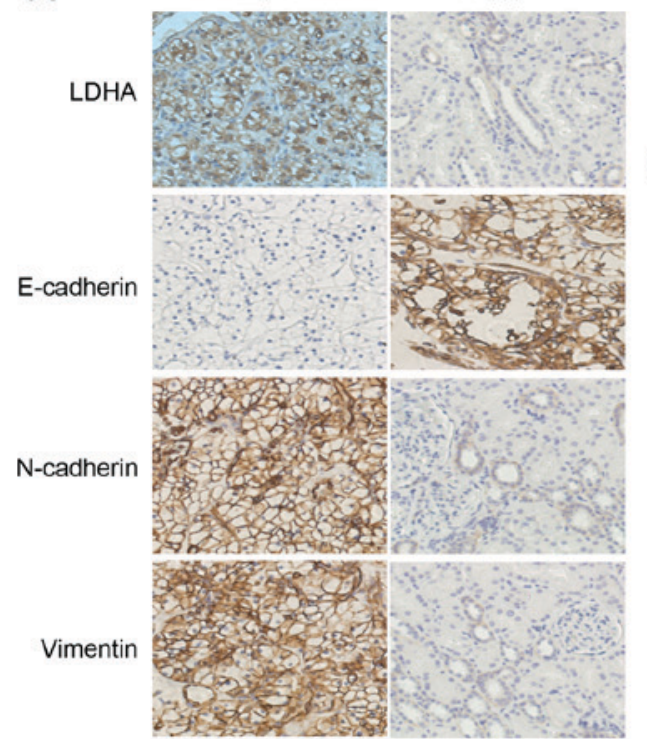

C

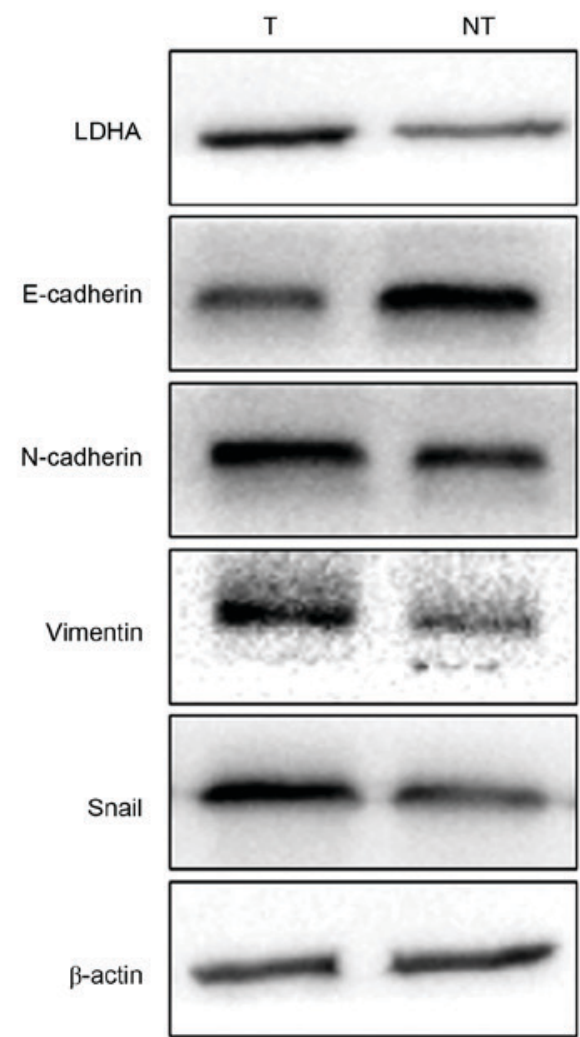

B
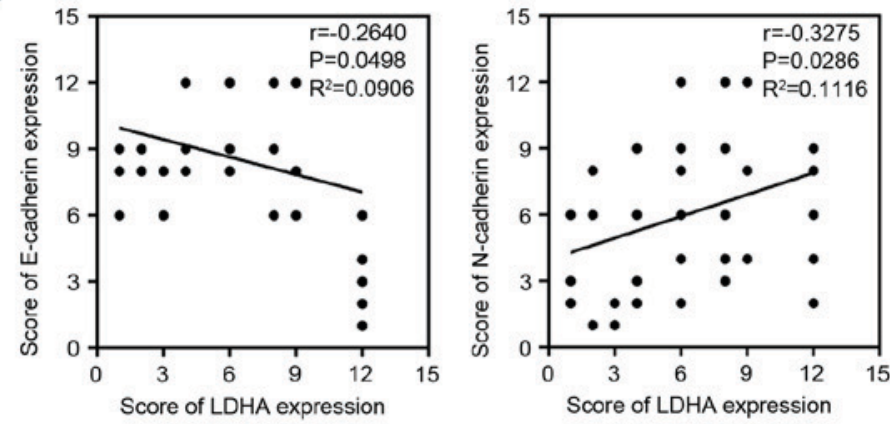

D LDHA

LDHA

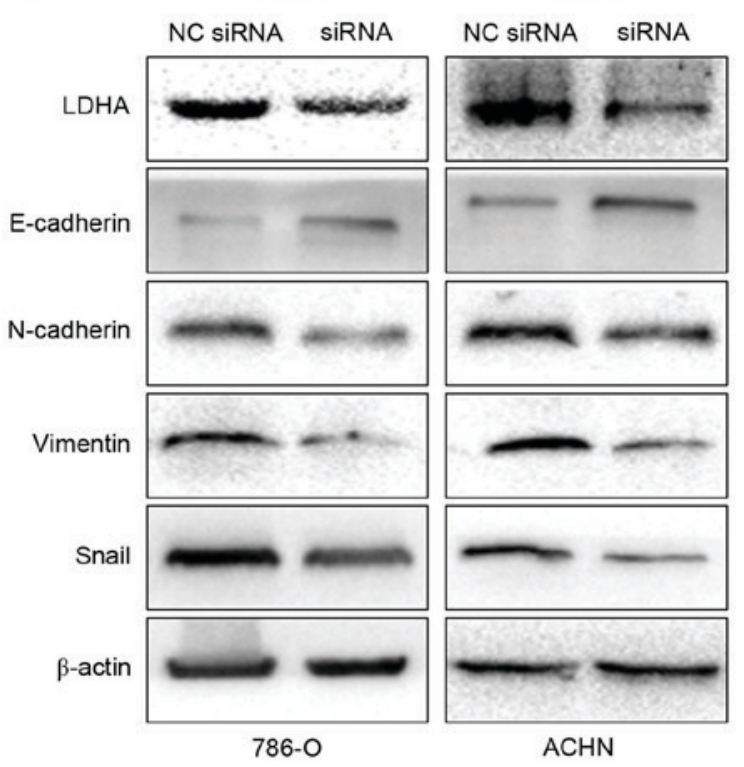

Figure 4. LDHA suppressed epithelial phenotype and promoted mesenchymal transition. (A-C) Immunohistochemical staining (magnification, x200) and western blotting showed that high expression of LDHA correlated with low expression of E-cadherin and high expression of $\mathrm{N}$-cadherin and vimentin. (D) Downregulation of LDHA expression inhibited the expression of E-cadherin and promotes the expression of N-cadherin, vimentin and Snail. LDHA, lactate dehydrogenase A; T, tumor tissue; NT, non-tumor tissue; siRNA, small interfering RNA; NC, negative control.

urgent to explore the molecular mechanism of RCC progression and discover the novel RCC markers and target agents.

Gradually acquiring a number of genetic mutations is necessary for the malignant transformation of cells. LDHA, a key enzyme in aerobic glycolysis, has a primary function to maintain the rapid regeneration of $\mathrm{NAD}^{+}(28)$. A growing body of evidence has suggested that LDHA was overexpressed and associated with poor prognosis of various cancers including pancreatic cancer, breast cancer and ovarian cancer $(29,30)$.
In the current study, on the basis of gathered specimens from RCCs and the relevant clinical information, the authors detected that LDHA was overexpressed in RCC tissues significantly and predicted worse survival following renal resection, which was in agreement with published observations (31). These data supported that LDHA could function as an oncogenic protein in RCC.

Tumor cells are characterized by increased glucose uptake, and increased lactate production, which are known 

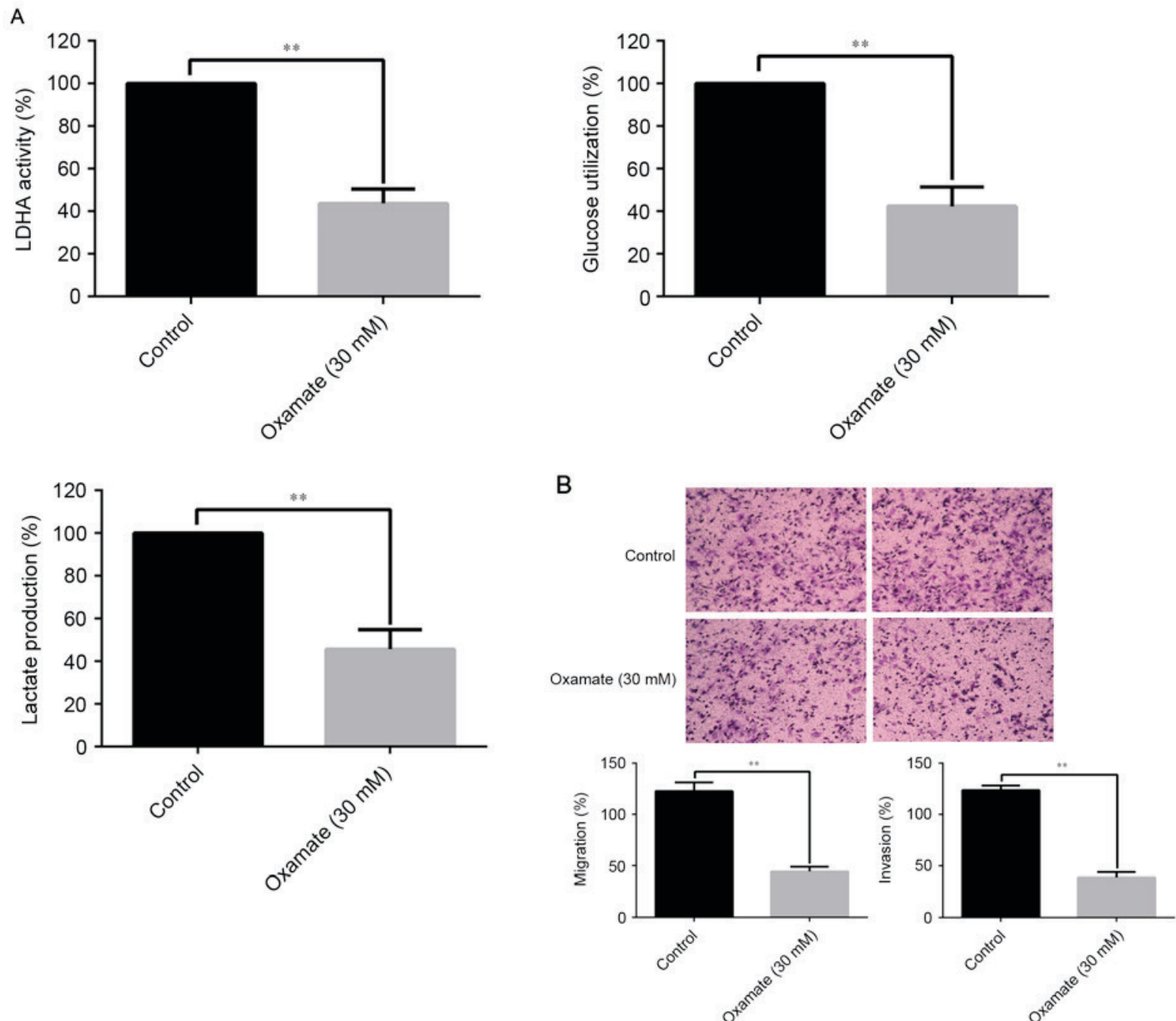

C
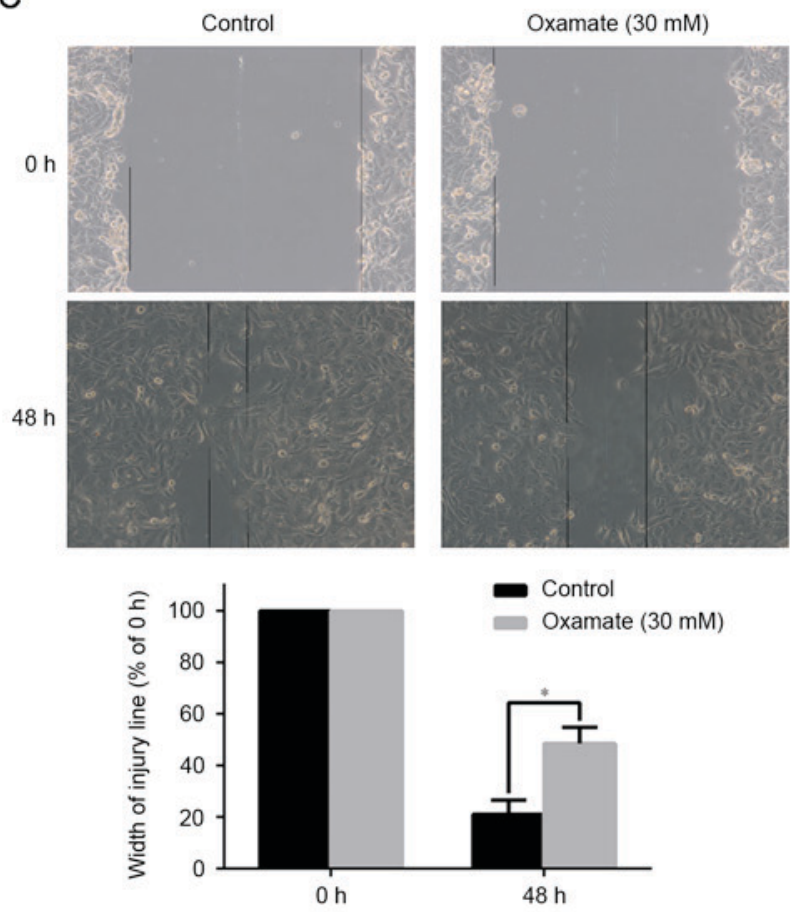

Figure 5. Oxamate suppressed tumor metastasis by inhibiting LDHA activity and epithelial-mesenchymal transition. (A) Results showed that oxamate significantly decreased the LDHA activity, glucose utilization and lactate production of RCC cells compared with the control group. (B) The migration and invasion ability of RCC cells treated with oxamate were decreased by Transwell assay (magnification, x100). (C) The migration ability of RCC cells treated with oxamate was decreased by wound healing assay (magnification, $\mathrm{x} 200$ ). Data are presented as mean \pm standard deviation. ${ }^{*} \mathrm{P}<0.05$, ${ }^{* * *} \mathrm{P}<0.01$ as indicated. LDHA, lactate dehydrogenase A; RCC, renal cell carcinoma. 
as the Warburg effect. In tumor cells, lactate is exported by monocarboxylate transporters leading to the acidification of microenvironment, however this alternation results in cell death in normal cells (32). Numerous attempts have been made to explore the changes of gene related with the Warburg effect (33). In the present research, a decreased level of consumption of glucose and production of lactate were observed as a response to downregulation of LDHA, which implied that LDHA may trigger aggressive forms of malignancy via regulating the Warburg effect. But the role of the change of the Warburg effect has not been illuminated.

Tumor metastasis is a primary factor affecting the prognosis of RCC patients (1). Therefore, it is critical to elucidate the mechanism of metastasis of RCC. The transition of epithelial cells to a mesenchymal phenotype, is the EMT (22). The role of EMT contributing to the metastatic potential of tumors has been widely accepted $(26,34)$. A recent study showed that EMT played a crucial role in RCC development and progression, it could be a therapeutic target in RCC patients (35). Multiple intracellular signaling pathways have been implicated in mediating EMT. Wang et al (36) showed that downregulation of LDHA would suppress tumor metastasis by downregulating the expression of matrix metalloproteinase (MMP)-9, MMP-2 and vascular endothelial growth factor. Therefore, the authors hypothesized that EMT might be implicated in the metastasis induced by the factor LDHA. In the present investigation, the authors firstly confirmed that LDHA level was positively related to tumor metastasis by clinical data analysis. Further studies showed that LDHA expression has a negative correlation with epithelial phenotype and a positive correlation with mesenchymal phenotype. It is worthwhile to note that LDHA was positively associated with Snail, a transcription factor regulating EMT. Furthermore, LDHA expression was silenced by LDHA-siRNA in RCC cells and obtained a reversed EMT phenotype including lower cell mobility, weaker invasion capacity, more epithelial maker expression and less expression of mesenchymal markers, which was in concert with the IHC result. Besides, experiments in vivo further confirmed the role of LDHA in facilitating RCC metastasis. These data strongly suggest that the dysregulation of LDHA is responsible for the initiation of EMT in RCC.

To establish the potential role of LDHA in clinical application and corroborate these data further, the authors extended their studies with treating LDHA inhibitor oxamate to the RCC cells, of which results indicated that oxamate could inhibit the activity of LDHA, the utilization of glucose and the production of lactate. Oxamate can also suppress the migration and invasion of RCC cells by affecting the EMT process. In summary, the study indicated that the high level of LDHA can promote tumor metastasis by stimulating initiation of EMT in renal cell carcinoma. Based on the combination of these data, the authors believe that LDHA would have therapeutic implications. However, the molecular mechanisms need further to fully elucidated.

\section{References}

1. Ljungberg B, Campbell SC, Choi HY, Jacqmin D, Lee JE, Weikert S and Kiemeney LA: The epidemiology of renal cell carcinoma. Eur Urol 60: 615-621, 2011.
2. Ferlay J, Shin HR, Bray F, Forman D, Mathers C and Parkin DM: Estimates of worldwide burden of cancer in 2008: GLOBOCAN 2008. Int J Cancer 127: 2893-2917, 2010.

3. Kabaria R, Klaassen Z and Terris MK: Renal cell carcinoma: Links and risks. Int J Nephrol Renovasc Dis 9: 45-52, 2016.

4. Moch H, Artibani W, Delahunt B, Ficarra V, Knuechel R, Montorsi F, Patard JJ, Stief CG, Sulser T and Wild PJ: Reassessing the current UICC/AJCC TNM staging for renal cell carcinoma. Eur Urol 56: 636-643, 2009.

5. Bilim V, Ougolkov A, Yuuki K, Naito S, Kawazoe H, Muto A, Oya M, Billadeau D, Motoyama T and Tomita Y: Glycogen synthase kinase-3: A new therapeutic target in renal cell carcinoma. Br J Cancer 101: 2005-2014, 2009.

6. Warburg O: On the origin of cancer cells. Science 123: 309-314, 1956.

7. Yuen CA, Asuthkar S, Guda MR, Tsung AJ and Velpula KK: Cancer stem cell molecular reprogramming of the Warburg effect in glioblastomas: A new target gleaned from an old concept. CNS Oncol 5: 101-108, 2016.

8. Taniguchi K, Sakai M, Sugito N, Kumazaki M, Shinohara H, Yamada N, Nakayama T, Ueda H, Nakagawa Y, Ito Y, et al: PTBP1-associated microRNA-1 and -133b suppress the Warburg effect in colorectal tumors. Oncotarget 7: 18940-18952, 2016.

9. Soltysova A, Breza J, Takacova M, Feruszova J, Hudecova S, Novotna B, Rozborilova E, Pastorekova S, Kadasi L and Krizanova O: Deregulation of energetic metabolism in the clear cell renal cell carcinoma: A multiple pathway analysis based on microarray profiling. Int J Oncol 47: 287-295, 2015.

10. Lim HY, Yip YM, Chiong E, Tiong HY, Halliwell B, Esuvaranathan K and Wong KP: Metabolic signatures of renal cell carcinoma. Biochem Biophys Res Commun 460: 938-943, 2015.

11. Goldman RD, Kaplan NO and Hall TC: Lactic dehydrogenase in human neoplastic tissues. Cancer Res 24: 389-399, 1964.

12. Sun X, Sun Z, Zhu Z, Guan H, Zhang J, Zhang Y, Xu H, and Sun M: Clinicopathological significance and prognostic value of lactate dehydrogenase A expression in gastric cancer patients. PloS One 9: e91068, 2014.

13. Xian ZY, Liu JM, Chen QK, Chen HZ, Ye CJ, Xue J, Yang HQ, Li JL, Liu XF and Kuang SJ: Inhibition of LDHA suppresses tumor progression in prostate cancer. Tumour Biol 36: 8093-8100, 2015.

14. Qiu H, Jackson AL, Kilgore JE, Zhong Y, Chan LL, Gehrig PA, Zhou C and Bae-Jump VL: JQ1 suppresses tumor growth through downregulating LDHA in ovarian cancer. Oncotarget 6: 6915-6930, 2015.

15. Yao F, Zhao T, Zhong C, Zhu J and Zhao H: LDHA is necessary for the tumorigenicity of esophageal squamous cell carcinoma. Tumour Biol 34: 25-31, 2013.

16. Fantin VR, St-Pierre J and Leder P: Attenuation of LDH-A expression uncovers a link between glycolysis, mitochondrial physiology, and tumor maintenance. Cancer Cell 9: 425-434, 2006.

17. Girgis H,MasuiO,WhiteNM,Scorilas A,RotondoF,Seivwright A, Gabril M, Filter ER, Girgis AH, Bjarnason GA, et al: Lactate Dehydrogenase A is a potential prognostic marker in clear cell renal cell carcinoma. Mol Cancer 13: 101, 2014.

18. Wang X, Xu L, Wu Q, Liu M, Tang F, Cai Y, Fan W, Huang H and Gu X: Inhibition of LDHA deliver potential anticancer performance in renal cell carcinoma. Urol Int: 2016.

19. Thiery JP and Sleeman JP: Complex networks orchestrate epithelial-mesenchymal transitions. Nat Rev Mol Cell Biol 7: 131-142, 2006.

20. Cao J, Wang H, Chen F, Fang J, Xu A, Xi W, Zhang S, Wu G and Wang Z: Galangin inhibits cell invasion by suppressing the epithelial-mesenchymal transition and inducing apoptosis in renal cell carcinoma. Mol Med Rep 13: 4238-4244, 2016.

21. Giannelli G, Koudelkova P, Dituri F and Mikulits W: Role of epithelial to mesenchymal transition in hepatocellular carcinoma. J Hepatol 65: 798-808, 2016.

22. NakazawaMandKyprianou N:Epithelial-mesenchymal-transition regulators in prostate cancer: Androgens and beyond. J Steroid Biochem Mol Biol 166: 84-90, 2017.

23. Smith BN and Bhowmick NA: Role of EMT in metastasis and therapy resistance. J Clin Med 5: E17, 2016.

24. Piva F, Giulietti M, Santoni M, Occhipinti G, Scarpelli M, Lopez-Beltran A, Cheng L, Principato G and Montironi R: Epithelial to mesenchymal transition in renal cell carcinoma: Implications for cancer therapy. Mol Diagn Ther 20: 111-117, 2016. 
25. Jiang F, Ma S, Xue Y, Hou J and Zhang Y: LDH-A promotes malignant progression via activation of epithelial-to-mesenchymal transition and conferring stemness in muscle-invasive bladder cancer. Biochem Biophys Res Commun 469: 985-992, 2016.

26. Chen Y, Sun Y, Rao Q, Xu H, Li L and Chang C: Androgen receptor (AR) suppresses miRNA-145 to promote renal cell carcinoma (RCC) progression independent of VHL status. Oncotarget 6: 31203-31215, 2015.

27. Hatzivassiliou G, Zhao F, Bauer DE, Andreadis C, Shaw AN Dhanak D, Hingorani SR, Tuveson DA and Thompson CB: ATP citrate lyase inhibition can suppress tumor cell growth. Cancer Cell 8: 311-321, 2005

28. Kaplan NO: Lactate dehydrogenase-structure and function. Brookhaven Symp Biol 17: 131-153, 1964.

29. Weide B, Elsasser M, Buttner P, Pflugfelder A, Leiter U, Eigentler TK, Bauer J, Witte M, Meier F and Garbe C: Serum markers lactate dehydrogenase and S100B predict independently disease outcome in melanoma patients with distant metastasis. Br J Cancer 107: 422-428, 2012.

30. Brown JE, Cook RJ, Lipton A and Coleman RE: Serum lactate dehydrogenase is prognostic for survival in patients with bone metastases from breast cancer: A retrospective analysis in bisphosphonate-treated patients. Clin Cancer Res 18: 6348-6355, 2012.
31. Girgis H,MasuiO,WhiteNM,Scorilas A,RotondoF,Seivwright A, Gabril M, Filter ER, Girgis AH, Bjarnason GA, et al: Lactate dehydrogenase $\mathrm{A}$ is a potential prognostic marker in clear cell renal cell carcinoma. Mol Cancer 13: 101, 2014.

32. Han T, Kang D, Ji D, Wang X, Zhan W, Fu M, Xin HB and Wang JB: How does cancer cell metabolism affect tumor migration and invasion? Cell Adh Migr 7: 395-403, 2013.

33. Sanders E and Diehl S: Analysis and interpretation of transcriptomic data obtained from extended Warburg effect genes in patients with clear cell renal cell carcinoma. Oncoscience 2: 151-186, 2015.

34. Garber K: Energy deregulation: Licensing tumors to grow. Science 312: 1158-1159, 2006.

35. Sanders E and Diehl S: Analysis and interpretation of transcriptomic data obtained from extended Warburg effect genes in patients with clear cell renal cell carcinoma. Oncoscience 2: 151-186, 2015.

36. Wang X, Xu L, Wu Q, Liu M, Tang F, Cai Y, Fan W, Huang H and $\mathrm{Gu} \mathrm{X}$ : Inhibition of LDHA deliver potential anticancer performance in renal cell carcinoma. Urol Int, 2016. 